\title{
Chronic Inflammatory Demyelinating Polyneuropathy
}

\author{
Capt H Smith \\ MB, BS, RAMC \\ Senior House Officer
}

Maj I Salam

$M B, B S, M R C P, R A M C$

Senior Registrar

Dr L J Findley
TD, MD, FRCP
Consultant Neurologist

Queen Elizabeth Military Hospital, Stadium Road, Woolwich, London SE184QH

SUMMARY: Chronic Inflammatory Demyelinating Polyneuropathy (CIDP) is a rare neurological disease. Definitive therapy is not yet established. We report our use of intravenous Immunoglobulin (iv IgG) in two cases of CIDP and review the therapeutic options available. Intravenous IgG is a ubiquitous practical treatment particularly in patients who do not respond to steroids and where a rapid response is required.

\section{Introduction}

CIDP is symmetrical sensorimotor neuropathy that follows a chronic course which may be progressive or relapsing in nature. Essentially this condition can be distinguished from Guillain-Barre Syndrome as the weakness in CIDP progresses over a minimum of 8 weeks(1).

Its prevalence is estimated at $2 / 100,000$ of the population(2). Less than a third of the patients give a history of vaccination or illness in the 6 weeks preceding the onset of symptoms. Pregnancy is a significant risk factor for relapse(3). There are no significant genetic markers(2).

The cause of the disease is unknown. Histopathologically peripheral nerves show demyelination and inflammatory infiltrates with $T$ cells being dominant(4). Histological similarity to Guillain-Barre Syndrome raises the possibility of a humoral factor producing an abnormality of the immune response(5).

The majority of patients present with mixed motor and sensory deficits. Sixteen percent may show cranial nerve involvement(3). Typical electrophysiological changes are found and $90 \%$ show a raised CSF protein level(6).

Three therapeutic options are available: corticosteroids, plasma exchange (PE) or iv IgG. The majority require maintenance therapy. They vary in the time taken for a response to be shown(7).

Two cases of CIDP were diagnosed in 1993 at Queen Elizabeth Military Hospital (QEMH) which are described below.

\section{Case 1}

A 62 year old female hospital cleaner presented with a 3 year history of paraesthesia in her lower limbs resulting in multiple falls. Though initially progressive in nature the symptoms were stable for 6 months prior to presentation.
There was no significant past medical history. She did not drink alcohol or smoke and had no significant family history of neurological disease. She was not on any med-o ication.

On examination there was no loss of muscle power in her limbs. Lower limbs were areflexic. There was loss of pain, temperature sensation and proprioreception up to the level of the knees bilaterally. Romberg's test was strongly positive. She had a high stepping gait and was unable to perform heel to toe test.

In addition to routine bloods the following investiga tions were within normal limits: VITB12, Folate, Autoimmune Antibodies, Ace Level, Serum and Urine Electrophoresis, CSF examination. Nerve conduction studies in the lower limbs showed significant slowing of motor velocities and loss of sensory action potentials consistent with a diagnosis of demyelinating polyneuropathy.

On clinical and electrophysiological criteria the diagnosis of CIDP was made. She was initiated on a 1 week course of Methylprednisolone $125 \mathrm{mg}$ daily followed by 2 weeks of alternate day therapy. Five months after this she had not shown any response. At this stage she was admitted and treated with a 5 day course of iv IgG ( $35 \mathrm{~g}$ per day in divided doses) without any side effects.

This resulted in rapid, significant improvement as assessed by lower limb coordination. The patient was then able to walk normally. On review 6 weeks after treatment the improvement had continued with the patient able to walk heel to toe along a narrow beam. At that time nerve conduction studies were unaltered.

\section{Case 2}

A 23 year old mother based in Cyprus presented 7 weeks after delivery of a healthy child with an 8 week history of progressive limb weakness. 
Initially she noted paraesthesia of her hands with difficulty in fine movements. Her symptoms progressed preventing her from carrying out routine household jobs and child care. At the time of presentation she was unable to stand from a chair. She had no other neurological symptoms.

Fourteen months earlier she had suffered a similar episode of progressive limb weakness lasting 6 months before partially resolving. At its nadir she could not climb stairs and it left her unable to run as a residual deficit.

She was a nonsmoker with no alcohol intake. She was not on medication. There was no family history of neurological disease.

On examination there was symmetrical distal wasting in all 4 limbs. Power in all limbs was grade 4 (MRC) in proximal muscle groups and grade 2 in distal groups. Reflexes were present in the upper limbs but absent in the lower. There was no deficit to any modality of sensation. Her gait was highstepping with normal co-ordination. The rest of her clinical examination was normal.

Investigations were as detailed for the first case. Her CSF protein was raised at $1.45 \mathrm{~g} / \mathrm{L}$ (normal up to $0.55 \mathrm{~g} / \mathrm{L}$ ). Nerve conduction studies showed a marked slowing of motor and sensory conduction consistent with severe demyelinating polyneuropathy.

A diagnosis of CIDP was made. Treatment was initiated with iv IgG (26g per day in divided doses). After a 5 day course the patient reported improvement. Objective power testing showed a return to near grade 5 power in all muscle groups.

On review 6 weeks after treatment her improvement in power on formal testing was maintained. CSF protein had dramatically decreased to $0.75 \mathrm{~g} / \mathrm{L}$ and the nerve conduction studies although still abnormal had significantly improved.

\section{Discussion}

To confirm the diagnosis of CIDP it is essential to show electrophysiological changes consistent with demyelination and to exclude acquired causes of this e.g. underlying malignancies, metabolic disorders, dysproteinaemias, inflammatory disorders and a vasculitic process. Hereditary causes must also be excluded(8).

The prognosis is worse than in Guillain-Barre: mortality is $10 \%$, only $5 \%$ of patients recover fully, and $85 \%$ have disability if left untreated(1).

Presently there are three therapeutic options. High dose corticosteroids in a dose of $100 \mathrm{mg}$ Prednisolone daily for 3 weeks followed by alternate day therapy until response occurs followed by a gradual decrease in dosage to a maintenance dose if required. The average time to response is 2 months with the full response being achieved after 6 months. Ninety-five percent will initially respond but $47 \%$ require maintenance steroid - a quarter of these will experience serious side effects on long term therapy(9). Azathioprine combined with steroid has not been shown to be superior in initial treatment to steroid alone. It is used however to allow reduction of the maintenance steroid dose(10). The use of other immunosuppressive agents like cyclophosphamide and Cyclosporin $\mathrm{A}_{\Omega}^{\circ}$ may be indicated in patients unresponsive to corticos-음 teroids, PE, and Iv IgG(2).

Plasma exchange is effective in $75 \%$ of cases $(5) . \stackrel{\overrightarrow{\vec{\rho}}}{\stackrel{\vec{\rho}}{+}}$ Remission however has to be maintained in most cases with three weekly exchanges(7). This is expensive, inva- $\frac{\bar{\sigma}}{\bar{*}}$ sive and only available in limited centres(2). A standard $\frac{\bar{C}}{\vec{D}}$ regime will involve $5 \%$ of body weight being exchanged $\stackrel{\Phi}{\complement}$ over 5 days for $4.5 \%$ human albumin. This involves insertion of a central line and a stay in an Intensive Care $\vec{O}$ Unit for monitoring. The cost for a single exchange is approximately $£ 3000(11)$. Side effects reported include $\vec{\omega}$ anaemia and complications resulting from central line? insertion. Plasma exchange is contraindicated in patients with cardiovascular instability and in those with poor venous access $(12)$.

The clinical and neurophysiological benefits of treat- 웅 ment with iv IgG have been established(13). Therapy results in improvement in $60 \%$ of cases. One third enter a remission for at least 3 years and may not require futuf treatment and the remaining responders require mainte $\vec{C} \vec{c}$ nance therapy. Improvement is seen within 5 days in mogst cases with a peak within 1 month(14). The most effectipe regime for maintenance therapy has been at a reduc $\theta$ dose at intervals of 2 weeks. The standard regime consisfs? of $0.4 \mathrm{~g} / \mathrm{kg}$ of iv IgG via a peripheral line daily forg days(15). A rare side effect is anaphylactic shock. The it: tial dose should be given slowly; once safety is proven t daily dose can then be given over 2 hours. Dependent on body weight the cost of a 5 day course is between $£ 2500 \stackrel{\odot}{\propto}$ and $£ 3000(16)$. Commercial preparations of iv IgG are $\overrightarrow{\vec{A}}$ readily available. These preparations exert an effect on $\frac{0}{3}$ cell mediated immunity directly by transfused antibodies but also by immunologically active proteins that are con-o tained in varying quantities in the commercial preparations such as Interferon(17). A randomised trial comparing iv IgG in Guillain-Barre syndrome concluded that this therapy is as effective as PE and may be superior(18).

Corticosteroids are the cheapest treatment but have the slowest onset and most significant long term side effects. They are the first line treatment in mild disease but in $\frac{5}{3}$ refractory or severe disease especially when a rapid response is needed they are ineffective. Iv IgG is already being used in some centres as a first line agent in disabling CIDP. It is no more expensive than plasma exchange and can be given to a wider range of patients $\frac{\vec{O}}{0}$ conveniently in all hospitals(1).

\section{Acknowledgements}

We wish to thank Lt Col J Dickinson for permission to report one of his patients and Brig G Ratcliffe for his helpful comments. 


\section{REFERENCES}

1. P J DYCK, A C LAIS . Chronic inflammatory polyradiculoneuropathy. Mayo Clin Proc 1975; 50: 621-637.

2. MCleod J G , Pollard J D. Recent advances in clinical neurology 1992; 7: 155-174.

3. MCCOMBe P A, Pollard J D. Chronic inflammatory demyelinating polyradiculoneuropathy. Brain 1987; 110: $1617-1630$.

4. Prineas J W, Mcleod J G. Chronic relapsing polyneuritis. J Neurol Sci 1976; 27:427-458.

5. DYCK P J, JASPER M D. Plasma exchange in chronic inflammatory demyelinating polyradiculoneuropathy. N Engl J Med 1986; 314: 461-465.

6. DyCK P J, LAIS A C. Chronic inflammatory polyradiculoneuropathy. Mayo Clin Proc 1975; 50: 621-637.

7. Pollard J D. Critical review of therapies in acute and chronic inflammatory demyelinating polyneuropathies. Muscle Nerve 1987; 10: 214-221.

8. LEWIS R A, SUMNer A J. The electrodiagnostic distinction between chronic familial and acquired demyelinative neuropathies. Neurology 1982; 32: 592-596.

9. DyCK P J, O'Brien P C. Prednisone improves chronic inflammatory demyelinating polyradiculoneuropathy more than no treatment. Ann Neurol 1982; 11: 136-141.
10. Dyck P J, O'BriEN P C. Combined azathioprine and prednisolone in chronic inflammatory demyelinating polyneuropathy. Neurology 1985 35: 1173-1176.

11. Dept Therapeutics. The National Hospital for Neurological disease. London.

12. DyCK P J, Benstead T J. Nonsystemic vasculitic neuropathy. Brain 1987; 110: 843-854.

13. Van de Merche, Vermeulen J. Chronic inflammatory demyelinating polyneuropathy. Brain 1989; 112: $1563-1571$.

14. Doorn P A van, Vermeuler M. Intravenous Immunoglobulin Treatment in Patients with Chronic के Inflammatory Demyelinating Polyneuropathy. Arch Neurol 1991; 48: 217-220.

15. DoORn P A vaN, VRAND A. High dose intravenous immunoglobulin treatment in chronic inflammatory demyelinating polyneuropathy. Neurology 1990; 40: 209-211.

16. British National Formulary. 1993.

17. LEE LAM, MCNICHOLL J . Immunologically active proteins in intravenous immunoglobin. Lancet 1993;

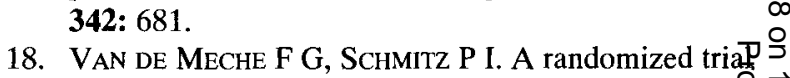
comparing intravenous immune globulin and plasm $\vec{C}$ exchange in Guillain-Barre syndrome. $N$ Engl $J$ Med 1992; 326 17: 1123-1129.

\section{The only way to check the vision of VDU operators is at their own workstation}

The Keeler Vutest has been designed to screen employees at their own workstation with minimum supervision in-house. It is an IBM compatible software driven system used to test the eyesight of VDU operators.

Increased productivity

* Testing takes place at employee's own workstation

* The test takes only 5 minutes to complete

* Testing on-site saves time and money

Minimum supervision

* Automatic printed results

* Comprehensive instructions for the employee

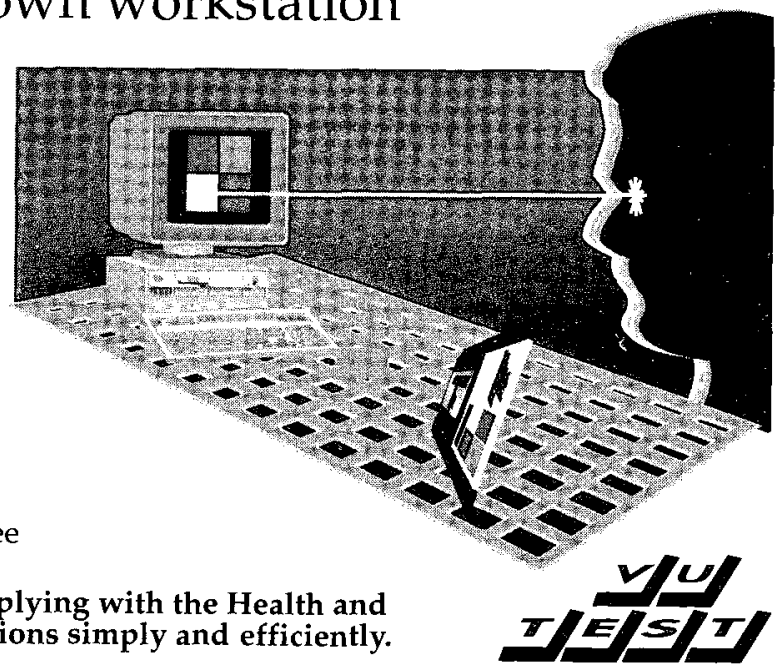

Vutest can ease the financial burden of complying with the Health and
Safety (Display Screen Equipment) Regulations simply and efficiently.

To receive further details please contact Keeler on Freephone 0800521251

VDU eye screening test

Keeler Ltd, Clewer Hill Road, Windsor, Berkshire SL4 4AA

Tel: 0753857177 Fax: 0753857817 